\title{
Vortex Entry and Nucleation of Antivortices in a Mesoscopic Superconducting Triangle
}

\author{
L. F. Chibotaru, ${ }^{1}$ A. Ceulemans, ${ }^{1}$ V. Bruyndoncx, ${ }^{2}$ and V. V. Moshchalkov ${ }^{2}$ \\ ${ }^{1}$ Afdeling Kwantumchemie, Katholieke Universiteit Leuven, \\ Celestijnenlaan 200F, B-3001 Leuven, Belgium \\ ${ }^{2}$ Laboratorium voor Vaste-Stoffysica en Magnetisme, Katholieke Universiteit Leuven, \\ Celestijnenlaan 200D, B-3001 Leuven, Belgium
}

(Received 24 July 2000)

\begin{abstract}
The nucleation of superconductivity in mesoscopic equilateral triangles is investigated by using the linearized Ginzburg-Landau equation (LGLE). The trigonal symmetry of the sample has a profound effect on the superconducting state in the presence of a magnetic field $H$ leading, in particular, to the formation of antivortices in symmetry-consistent states. For the same given irreducible representation, vortices enter always by three via the middle of the edges, approach the center, and then are dispatched towards the corners of the triangle. The measured superconducting phase boundary $T_{c}(H)$ is in good agreement with the $T_{c}(H)$ line found from the LGLE.
\end{abstract}

DOI: 10.1103/PhysRevLett.86.1323

PACS numbers: 74.60.Ec, 74.20.De, 74.25.Dw

The superconducting state in microcylinders and disks in an applied magnetic field nucleates in the form of the giant vortex state (GVS) [1-4]. The phase boundary in the $H-T$ plane [critical temperature vs field $T_{c}(H)$ ] shows oscillations corresponding to the cusplike $T_{c}(H)$ line formed by the states with different orbital quantum numbers. While the GVS appearance in these samples is well understood as a consequence of the rotational symmetry, the vortex states in samples of lower symmetry are still under investigation [5-7].

The rotational symmetry of the disk, $C_{\infty}$, can be obtained in the limit $N \rightarrow \infty$ of the polygons with rotational symmetry $C_{N}$, where $N$ is the number of vertices. Despite the high symmetry of the regular polygon samples, it is not easy to anticipate how the solutions for different vortex states will look in this case.

In this Letter, using the linearized Ginzburg-Landau equation (LGLE), the phase boundary $T_{c}(H)$, the properties of different vortex states, and vortex patterns evolution as a function of the applied field are investigated in a mesoscopic equilateral triangle. The validity of the new symmetry-consistent solutions is checked by comparing the calculated and the measured phase boundary for a mesoscopic Al triangle, although our approach remains valid for other homogeneous superconducting polygons (for square, see Ref. [8]). Remarkably, the presence of the $C_{N}$ symmetry of polygons leads to a spontaneous formation of antivortices, which appear to conserve the discrete symmetry imposed by the boundary conditions.

The nucleation of superconductivity is described via the solution of the LGLE [9]:

$$
\frac{1}{2 m^{*}}\left(-i \hbar \nabla-\frac{2 e}{c} \mathbf{A}\right)^{2} \psi=-\alpha \psi,
$$

where $\psi$ stands for the complex superconducting order parameter and $\alpha$ is the first Ginzburg-Landau parameter, related to the temperature-dependent coherence length, $\xi(T)$, by $\alpha=-\hbar^{2} / 2 m^{*} \xi^{2}(T)$. Despite the simple form, the solution of this equation is not trivial at all since the superconducting boundary conditions are imposed on $\psi$ in finite samples [9]:

$$
\left.\left(-i \hbar \nabla-\frac{2 e}{c} \mathbf{A}\right) \psi\right|_{n}=0 .
$$

It reduces to the Neumann boundary condition, $\left.\nabla \psi\right|_{n}=0$, when the magnetic vector potential, $\mathbf{A}$, can be chosen in a form with zero normal component at the boundary of the sample. Beside disks [10], such a choice has been achieved only for infinite slabs and wedges $[11,12]$.

We have developed an analytical gauge transformation for the vector potential, which gives $A_{n}=0$ along the boundary line of an arbitrary regular polygon. The details of the method will be given elsewhere [13]. With this choice of gauge, the problem reduces to an eigenvalue problem in a basis set of functions obeying Neumann boundary conditions. It is convenient to take for such a basis set the eigenfunctions of the zero field problem. Given the threefold rotational symmetry of the problem, the solutions of Eq. (1) are characterized by irreducible representations (irreps) of the cyclic group $C_{3}$. This group contains three irreps, $A, E_{-}$, and $E_{+}$, with the characters $\exp (i n \pi / 2), n=0,-1$, and 1 under the threefold rotation, respectively [14]. The unnormalized eigenfunctions of the zero field problem, i.e., of the particle in the equilateral triangular box obeying Neumann boundary conditions, can be obtained [13] in the following form for the irrep $A$ : 


$$
\begin{aligned}
\psi_{p q}^{A}(x, y)= & \cos \frac{\pi}{h}(2 p+q) y \cos \frac{\pi}{h} \sqrt{3} q x+\cos \frac{\pi}{h}(-p+q) y \cos \frac{\pi}{h} \sqrt{3}(p+q) x \\
& +\cos \frac{\pi}{h}(p+2 q) y \cos \frac{\pi}{h} \sqrt{3} p x, \quad p \geq q=0,1,2, \ldots, \\
\psi_{p q}^{A}(x, y)= & -\sin \frac{\pi}{h} \sqrt{3} q x \cos \frac{\pi}{h}(2 p+q) y+\sin \frac{\pi}{h} \sqrt{3}(p+q) x \cos \frac{\pi}{h}(-p+q) y \\
& -\sin \frac{\pi}{h} \sqrt{3} p x \cos \frac{\pi}{h}(p+2 q) y, \quad p \geq q=1,2, \ldots,
\end{aligned}
$$

where the first set is symmetric with respect to the vertical symmetry planes, and the second one antisymmetric. For the irreps $E_{+}$and $E_{-}\left[\psi^{E_{-}}=\left(\psi^{E_{+}}\right)^{*}\right]$ :

$$
\begin{aligned}
\psi_{p q}^{E_{-}}(x, y)= & \exp \left\{\frac{\pi i}{h}[(2 p+q) y-\sqrt{3} q x]\right\}+\exp \left\{\frac{\pi i}{h}[(p+2 q) y-\sqrt{3} p x]\right\} \\
& +\exp \left\{\frac{\pi i}{h}[(-p+q) y+\sqrt{3}(p+q) x]-2 \pi q i \pm \frac{2 \pi}{3} i\right\} \\
& +\exp \left\{\frac{\pi i}{h}[-(p+2 q) y-\sqrt{3} p x]+2 \pi p i \mp \frac{2 \pi}{3} i\right\} \\
& +\exp \left\{\frac{\pi i}{h}[(p-q) y+\sqrt{3}(p+q) x]-2 \pi p i \pm \frac{2 \pi}{3} i\right\} \\
& +\exp \left\{\frac{\pi i}{h}[-(2 p+q) y-\sqrt{3} q x]+2 \pi q i \mp \frac{2 \pi}{3} i\right\}
\end{aligned}
$$

where the two signs correspond to the following quantum numbers $(n=0,1,2, \ldots)$ :

$$
\begin{aligned}
& q=n+1 / 3, \quad p=q, q+1, \ldots, \text { upper sign, } \\
& q=n+2 / 3, \quad p=q, q+1, \ldots, \text { lower sign. }
\end{aligned}
$$

In Eqs. (3) and (4), $h=\sqrt{3} a / 2$ is the height, and $a$ the edge of the triangle. The above solutions are similar by their structure to the solutions of the corresponding "particle-in-the-box" problem with the $\psi=0$ boundary condition [15]. In the following calculations, for each irrep, a basis of only the 40 lowest eigenstates was used.

Figure 1 shows the results of our calculations of the lowest eigenstates. The lowest Landau level, which defines the superconducting phase boundary $T_{c}\left(\Phi / \Phi_{0}\right)$, shows an oscillatory cusplike behavior as a function of $\Phi / \Phi_{0}$, corresponding to the transitions between the states belonging to different irreps. This closely resembles the situation in a superconducting disk, with the important difference that the latter has the symmetry $C_{\infty}$ of a two-dimensional rotator with an infinite number of irreps (rotational quantum numbers) [1]. As a consequence, in the low-energy region of the spectrum there is no "repulsion" of levels in the disk, whereas for the equilateral triangle the solutions of the LGLE show a regular pattern of avoided crossings between levels belonging to the same irreps (Fig. 1a).

Figure $1 \mathrm{~b}$ shows the measured $T_{c}(H)$ phase boundary for the mesoscopic $\mathrm{Al}$ triangle. The samples were prepared by using electron beam lithography and lift-off techniques. The critical temperature $T_{c}$ is defined as the midpoint of the resistive transition, where superconducting fluctuations are unimportant. For more experimental details, we refer to Ref. [16]. The theoretical phase boundary is obtained (Fig. 1a) as the ground state level. The agreement between the calculated lowest Landau level and the measured $T_{c}(\Phi)$ is quite good, which justifies both the applicability of the LGLE and the truncation of the basis set used in the calculations. Especially the cusp positions coincide very well (thin vertical lines). The asymptotic behavior of the phase boundary line, at large flux, is in good agreement with the ratio of critical fields $H_{c 2} / H_{c 3}=0.41$ found for a $\pi / 3$ wedge [12].

Each smooth line element on the phase diagram $a^{2} / \xi^{2}(T)$ versus field $\left(\Phi / \Phi_{0}\right)$ corresponds to an integer winding number, $L$ (vorticity), related to the fluxoid quantization. This number is zero (no fluxoids) for the first line starting on the left (Fig. 1a) and increases by 1 upon each transition to the next ground state. In the case of a disk the vorticity is just the orbital quantum number, $L$, defining the flux, $L \Phi_{0}$, carried by the GVS $[1,3]$. Therefore in a disk, the symmetry-consistent solutions of the LGLE will correspond to the GVS ( $L>1)$ pinned by the rotational axis of the disk [1]. In the case of a triangle the rotational axis is of finite order and, therefore, the distribution of vortices in the symmetry-consistent solutions, considered here, is not a priori evident. Figure 2 shows the distribution of the density of the order parameter $|\psi|^{2}$ in the states with vorticity $L=0, \ldots, 3$. In general, $|\psi|^{2}$ increases near the corners of the triangle, which is in line with the known trend of nucleation of surface superconductivity $[5,6]$.

The density distribution $|\psi|^{2}$ is always in agreement with the $C_{3 v}$ symmetry of the equilateral triangle, which is higher than the $C_{3}$ symmetry of the LGL equation. This 


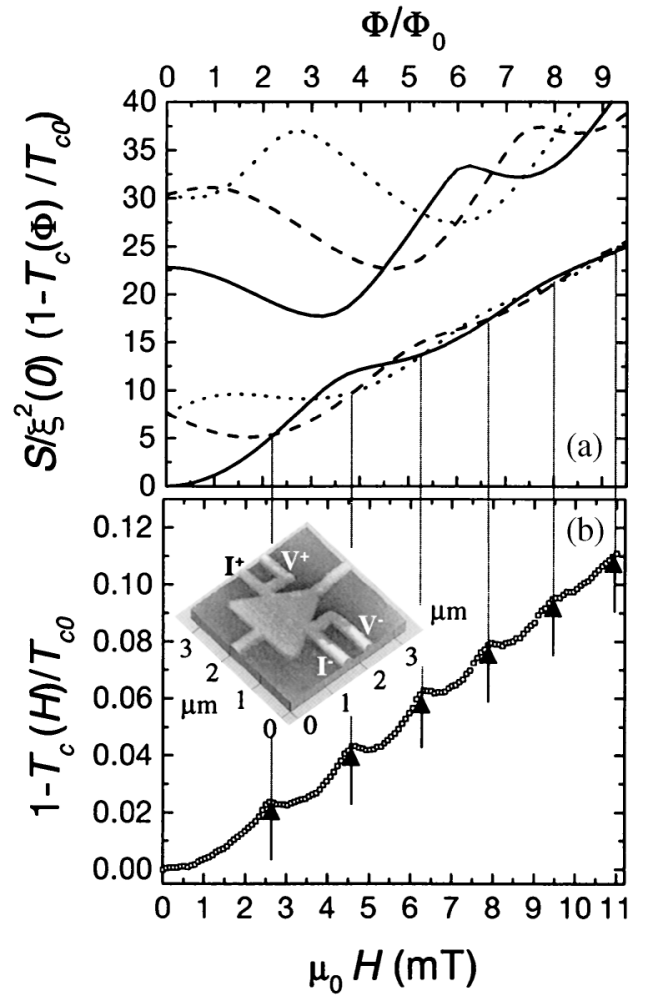

FIG. 1. (a) Calculated Landau levels for an equilateral triangle with boundary conditions given by Eq. (2). Continuous, dashed, and dotted lines correspond to states of symmetry $A, E_{+}$, and $E_{-}$. See the text for more details. (b) Experimental $T_{c}(H)$ phase boundary of an equilateral mesoscopic triangle of side length $a=1.9 \mu \mathrm{m}$ (open squares). The $T_{c}(H)$ data have been corrected for the presence of the measuring leads: a parabolic term arising from the $w=0.33 \mu \mathrm{m}$ wide contact lines was subtracted from $T_{c}(H)$. The arrows and the vertical dotted lines indicate the positions of the cusps in $T_{c}(H)$, as calculated from the LGL theory. In order to obtain a good correspondence for the experimental and the theoretical positions of the transitions $L \rightarrow L+1$ an effective sample area $S_{\text {eff }}=1.76 \mu \mathrm{m}^{2}$ has been used. The inset shows an atomic force microscopy (AFM) micrograph of the studied triangle. The $T_{c}(H)$ boundary is measured resistively, with the transport current fed through contacts $I^{+} / I^{-}$, and the voltage measured across the contacts $V^{+} / V^{-}$.

density is indeed invariant under the mirror reflections in vertical planes, contained in the group $C_{3 v}$, since these induce complex conjugation of the order parameter. In the case of small $L$ 's, vortices can occupy one central position and three positions on the lines from the center to the corners of the triangle. Integration of the gradient of phase of the order parameter along the contours encircling the vortices has shown that the vortices in corner positions are always $\Phi_{0}$ vortices. In contrast, the central vortex can have different winding numbers in order to adjust the total vorticity of a given state. The contribution of the two kinds of vortices (central + three corner) to the total winding number of the states, shown in Fig. 2, is given by

$$
L=n+3 m, \quad n=0,1,-1, \quad m=0,1 .
$$

The nature of the central vortex changes, whenever vorticity is changed by 1 . In the ground state the central vortex
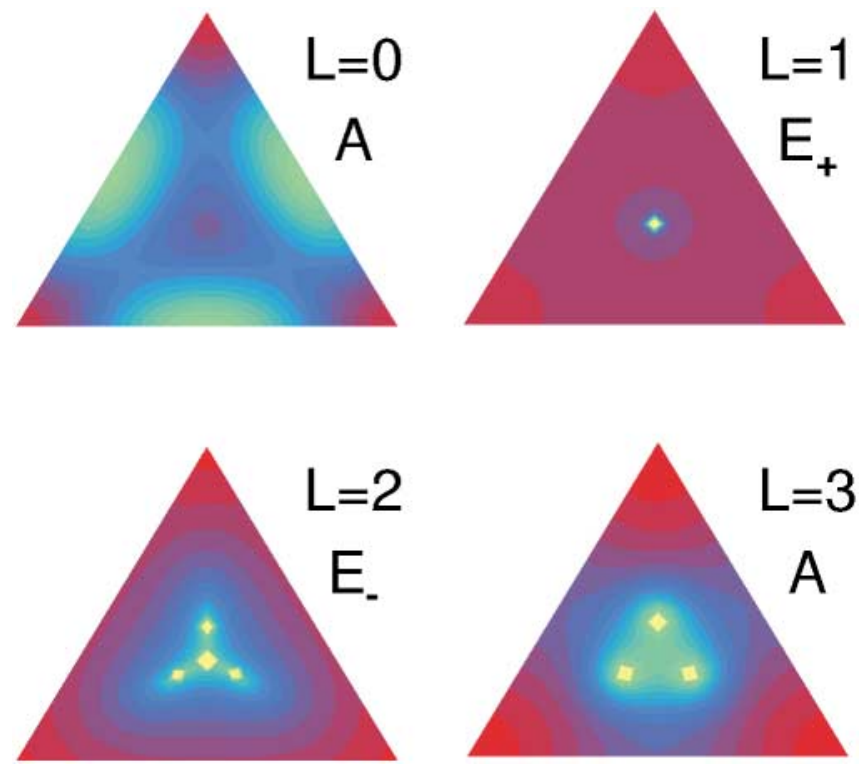

FIG. 2 (color). Density of the order parameter at the middle of the lowest four cusps in the $T_{c}(H)$ phase diagram in Fig. 1. The highest $|\psi|^{2}$ density is shown in red, followed by blue, and green. The lowest values, drawn in yellow, indicate the positions of vortices and antivortices. The panel for the $E_{-}$state, zoomed 16 times, shows the presence of an $\Phi_{0}$ antivortex in the center.

is absent in the $n=0$ state, it is a $\Phi_{0}$ vortex in the $n=1$ state, and it is an antivortex ( $-\Phi_{0}$ vortex) in the $n=-1$ state (see Fig. 2). The sequence of winding numbers of the central vortex $(-1,0,1)$ is periodically repeated when going from left to right. Note that such a relation between the winding numbers of the central vortex and the symmetry of the order parameter is already expected from the definition of the basis functions of irreps [14]. Actually, the states with winding numbers differing by multiples of three correspond to the same irrep. However the GVS does not arise in the lowest Landau level: because the kinetic energy of a vortex is proportional to $L^{2}$, the system prefers to split the giant vortex into a sum of smaller vortices [17] if there are no special symmetry restrictions. This explains why only three numbers mentioned above appear as winding numbers for the central vortex. On the other hand, the formation of antivortices is dictated by discrete symmetry. Indeed, in the state with $L=2$, one cannot put two $\Phi_{0}$ vortices on the equilateral triangle keeping the $C_{3 v}$ symmetry. The dilemma is solved by distributing three $\Phi_{0}$ vortices and one $\Phi_{0}$ antivortex.

Next we turn to the investigation of the flux patterns following one of the three continuous lines in the ground state (Fig. 1a). The solutions of Eq. (1), for a field sweep along eigenstates of the same symmetry, show that in all cases the flux enters by three $\Phi_{0}$ vortices through the edges centers. This flux entry obeys the $C_{3 v}$ symmetry constraints of the $|\psi|^{2}$ distribution, and moreover is physically justified because the density is the lowest just in these three points on the sides of the triangle. The entrance itself takes place exactly when the system passes over the top of an avoided crossing barrier (see Fig. 1a). Consecutively, the 

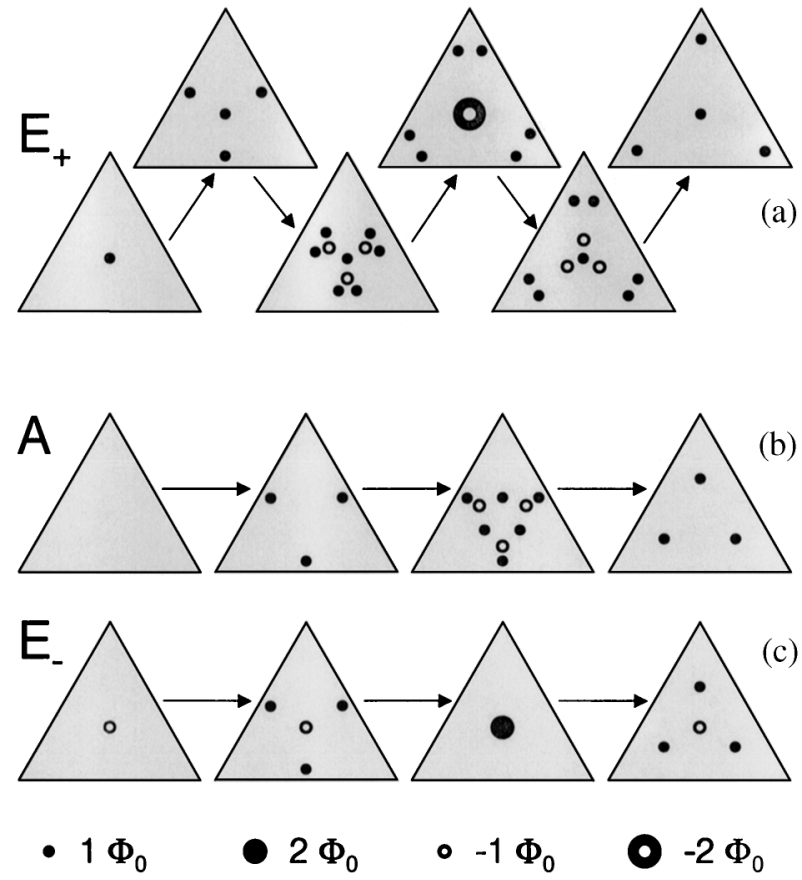

FIG. 3. Schematic plots showing different stages of the vortex entry for each given irrep: $L=4$ (a), 3 (b), and 2 (c). Filled and empty circles stand for vortices and antivortices, respectively.

vortices which entered laterally approach the center and then, at some specific value of the applied field, are transformed into three corner-directed vortices which are dispatched towards the corners, thus paving the way for the entrance of the next vortex triade. The stages of the vortex pattern evolution with increasing field are shown schematically in Fig. 3 for the three irreps. Most interestingly, the mechanism of reorientation of the entered vortices differs dramatically in the states of different symmetry. Thus in the $E_{+}$state each of the entered vortices deconvolutes into two $\Phi_{0}$ vortices and one $\Phi_{0}$ antivortex when approaching the center of the triangle; while the $\Phi_{0}$ vortices move in pairs towards the corners, the three antivortices annihilate with the central $\Phi_{0}$ vortex to form a $2 \Phi_{0}$ giant antivortex, which then splits back yielding the initial vortex in the center and three $\Phi_{0}$ antivortices dispatched to the corners; later on each of these antivortices meets two $\Phi_{0}$ vortices and recombines with them to a single $\Phi_{0}$ vortex. In the $A$ state, when the entered vortices approach the center of the triangle, the central region explodes into three $\Phi_{0}$ vortices and three $\Phi_{0}$ antivortices; while the antivortices annihilate in pairs with the entered vortices, the three $\Phi_{0}$ vortices from the central region move towards the corners of the triangle. Finally, the simplest scenario of the vortex reorientation is achieved in the $E_{-}$state: the entered vortices annihilate with the central antivortex resulting in a $2 \Phi_{0}$ giant vortex, which transforms into a $\Phi_{0}$ antivortex in the center and three $\Phi_{0}$ vortices dispatched to the corners of the triangle.

Local vortex imaging techniques, like scanning Hall probe [18], scanning tunneling [19], and magnetic force microscopy [20], are very promising for visualization of these predicted novel vortex patterns.

In conclusion, the nucleation of superconductivity in mesoscopic equilateral triangles was investigated. This nucleation is accompanied by the formation of the isolated vortices rather than a GVS. The formation of antivortices both in the stable vortex configurations and also at different stages of the evolution of the vortex patterns has been found. The antivortices originate from the competition between the infinite order rotational symmetry of the applied field and the finite order point group of the superconducting samples. Our results apply to a finite temperature interval close to $T_{c}$, where the effect of the nonlinear term in the Ginzburg-Landau equations can be neglected. At lower temperature the presence of the nonlinear term could give rise to symmetry-breaking effects.

We are grateful to G. Teniers for his help in preparing the figures, and to M. Cannaerts for the AFM measurement. The work was supported by the Concerted Action Scheme (GOA) of the Flemish Government, the Fonds voor Wetenschappelijk Onderzoek Vlaanderen (FWO), and by the ESF Programme VORTEX.

[1] D. Saint-James, Phys. Lett. 15, 13 (1965).

[2] H. J. Fink and A. G. Presson, Phys. Rev. 151, 219 (1966).

[3] V.V. Moshchalkov, X. G. Qiu, and V. Bruyndoncx, Phys. Rev. B 55, 11793 (1997).

[4] P. S. Deo, V. A. Schweigert, F. M. Peeters, and A. K. Geim, Phys. Rev. Lett. 79, 4653 (1997).

[5] V. M. Fomin, V. R. Misko, J. T. Devreese, and V. V. Moshchalkov, Solid State Commun. 101, 303 (1997).

[6] V. M. Fomin, V.R. Misko, J. T. Devreese, and V. V. Moshchalkov, Phys. Rev. B 58, 11703 (1998).

[7] V. A. Schweigert and F. M. Peeters, Phys. Rev. B 57, 13817 (1998); 60, 3084 (1999).

[8] L.F. Chibotaru, A. Ceulemans, V. Bruyndoncx, and V. V. Moshchalkov, Nature (London) 408, 833 (2000).

[9] P.-G. de Gennes, Superconductivity of Metals and Alloys (Benjamin, New York, 1966).

[10] R. B. Dingle, Proc. R. Soc. London A 211, 500 (1952).

[11] D. Saint-James, G. Sarma, and E. J. Thomas, Type II Superconductivity (Pergamon Press, Oxford, 1973).

[12] A. Houghton and F. B. McLean, Phys. Lett. 19, 172 (1965).

[13] L.F. Chibotaru, A. Ceulemans, V. Bruyndoncx, and V. V. Moshchalkov (to be published).

[14] L.D. Landau and E.M. Lifshitz, Quantum Mechanics (Pergamon, Oxford, 1975), 2nd ed.

[15] W.-K. Li and S. M. Blinder, J. Math. Phys. (N.Y.) 26, 2784 (1985).

[16] V. Bruyndoncx et al., Europhys. Lett. 36, 449 (1996).

[17] A. A. Abrikosov, Fundamentals of the Theory of Metals (North-Holland, Amsterdam, 1988).

[18] A. M. Chang et al., Appl. Phys. Lett. 61, 1974 (1992).

[19] H. F. Hess et al., Phys. Rev. Lett. 62, 214 (1989).

[20] A. Moser et al., Phys. Rev. Lett. 74, 1847 (1995). 